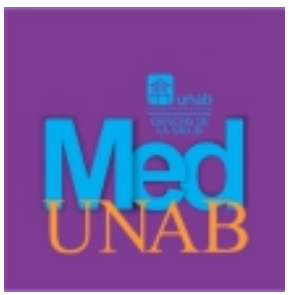

REVISTA DE LA FACULTAD

DE CIENCIAS DE LA SALUD

\title{
Epilepsia del lóbulo temporal: una revisión de tema sobre el abordaje diagnóstico
}

Temporal Lobe Epilepsy: a Topic Review on Diagnostic Approach

Epilepsia do lobo temporal: uma revisão sobre a abordagem diagnóstica

Ana Teresa Araujo-Reyes, MD., Esp. ${ }^{1}$, Josefina Sandoval, MD., Esp. ${ }^{2}$, Antonio Carrasco Ore, MD., Esp. ${ }^{3}$, María Alejandra Baquero-Serrano, MD. ${ }^{4}$

1. Médico especialista en Radiología e Imágenes Diagnósticas y Neuroradiología, Servicio de Radiología, Clínica FOSCAL. Bucaramanga, Colombia.

2. Médico especialista en Radiología e Imágenes Diagnósticas y Neuroradiología. Instituto de Neurología y Neurocirugía Manuel Velasco Suarez. México.

3. Médico especialista en Radiología e Imágenes Diagnósticas. Instituto Nacional de Ciencias Neurológicas. Lima, Perú.

4. Médica Residente de Radiología e Imágenes Diagnósticas, Universidad Autónoma de Bucaramanga, Bucaramanga, Colombia.

Correspondencia: Ana Teresa Araujo Reyes. Radiólogos Especializados de Bucaramanga S.A. Centro Médico Carlos Ardila Lülle-Torre A. Floridablanca, Santander, Colombia. Teléfono: 3173009692. Código Postal: 6810074.E-mail: araujoreyesanateresa@gmail.com

\section{INFORMACIÓN ARTÍCULO}

Artículo recibido: 27 de junio de 2018

Artículo aceptado: 29 de julio de 2019

DOI: https://doi.org/10.29375/01237047.3208

\section{RESUMEN}

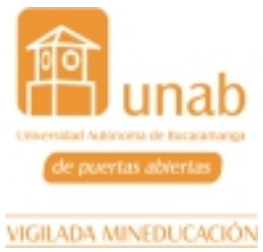

Introducción. Según la Organización Mundial de la Salud (OMS) la epilepsia constituye uno de los trastornos neurológicos más frecuentes en el mundo. Las crisis epilépticas se consideran una manifestación clínica originada por una descarga excesiva de neuronas a nivel cerebral. Su prevalencia es mayor en Latinoamérica y el Caribe que en países desarrollados; las crisis, en la mayoría de los casos, corresponden a la epilepsia del lóbulo temporal, cuya anormalidad anatómica más habitual es la esclerosis del hipocampo. El objetivo es exponer el protocolo básico de epilepsia de manera 
Cómo citar.

Araujo-Reyes AT, Sandoval J, Carrasco Ore A, Baquero-Serrano

MA. Epilepsia del lóbulo temporal: una revisión de tema sobre el abordaje diagnóstico. MedUNAB. 2019;22(2):228-241. doi:10.29375/01237047.3208 estandarizada y su utilidad en el planeamiento quirúrgico, y así, conociendo todas las herramientas estudiadas hasta el momento, se realice un adecuado y oportuno diagnóstico.

División de los temas tratados. Se realizó una revisión de tema de la literatura existente en las bases de datos Pubmed, Cochrane y Medline desde 1980 hasta 2018 acerca de la epilepsia del lóbulo temporal. Se expone de manera concisa la anatomía, fisiología, fisiopatología, presentación clínica y diagnóstico imaginológico de la patología.

Conclusiones. Esta patología se caracteriza por tener el foco epileptogénico en los lóbulos temporales con alta probabilidad de control con técnicas quirúrgicas cada vez menos agresivas. El diagnóstico es inducido a través de la clínica; sin embargo, las nuevas técnicas de imagen estructurales y funcionales son el método diagnóstico de elección en pacientes con sospecha de esta patología y, de esta manera, lograr la realización de un diagnóstico más certero y oportuno.

Palabras clave:

Epilepsia; Convulsiones; Lóbulo temporal; Espectroscopia de resonancia magnética; Terapéutica.

\section{ABSTRACT}

Introduction. According to the World Health Organization (WHO), epilepsy is one of the world's most frequent neurological disorders. Epileptic seizures are considered to be a clinical manifestation caused by an excessive discharge of neurons in the brain. Its prevalence is higher in Latin America and the Caribbean than in developed countries. In most cases, seizures correspond to temporal lobe epilepsy, whose most common anatomic abnormality is hippocampal sclerosis. The objective is to present the basic epilepsy protocol in a standardized form and its usefulness in surgical planning, in order to perform a correct and timely diagnosis, knowing all of the tools that have been studied until now.

Division of Covered Topics. A topic review of the existing literature in the Pubmed, Cochrane and Medline databases from 1980 to 2018 was performed on temporal lobe epilepsy. The anatomy, physiology, physiopathology, clinical presentation and imaging diagnosis of the pathology are concisely presented.

Conclusions. This pathology is characterized by having an epileptogenic focus in the temporal lobes, with a high probability of control with surgical techniques that are becoming less aggressive. Diagnoses are made through clinics. However, new structural and functional imaging techniques are the diagnosis method of choice for patients suspected to have this pathology in order to make a more accurate and timely diagnosis.

Keywords: Epilepsy; Seizures; Temporal Lobe; Magnetic Resonance Spectroscopy; Therapeutics.

\section{RESUMO}

Introdução. Introdução. Segundo a Organização Mundial da Saúde (OMS) a epilepsia é um dos distúrbios neurológicos mais frequentes do mundo. As crises epilépticas são consideradas uma manifestação clínica causada por uma descarga excessiva de neurônios no nível cerebral. Sua prevalência é maior na América Latina e no Caribe do que nos países desenvolvidos; as crises, na maioria dos casos, correspondem à epilepsia do lobo temporal, cuja anormalidade anatômica mais comúm é a esclerose do hipocampo. O objetivo deste trabalho é expor o protocolo básico de epilepsia de forma padronizada e sua utilidade no planejamento cirúrgico, e assim, conhecendo todas as ferramentas estudadas até o momento, seja feito um diagnóstico adequado e oportuno. Divisão dos tópicos discutidos. Foi realizada uma revisión da literatura existente nas bases de dados Pubmed, Cochrane e Medline desde 1980 até 2018 sobre a epilepsia do 
lobo temporal. Apresenta concisamente a anatomia, fisiologia, fisiopatologia, apresentação clínica e diagnóstico por imagem da patologia. Conclusão. A patologia é caracterizada por ter o foco epileptogênico nos lobos temporais, com alta probabilidade de controle, com técnicas cirúrgicas cada vez menos agressivas. O diagnóstico é induzido pela clínica; no entanto, as novas técnicas de imagem estruturais e funcionais são o método diagnóstico de maior escolha em pacientes com suspeita dessa patologia e, assim, conseguem um diagnóstico mais preciso e oportuno.

Palavras-chave:

Epilepsia; Convulsões; Lobo temporal; Espectroscopia de ressonância magnética; Terapêutica.

\section{Introducción}

Según la OMS, la epilepsia constituye uno de los trastornos neurológicos más frecuentes en el mundo. Se estima que una de cada cien personas tiene epilepsia; en el mundo la padecen más de 70 millones de personas, de las cuales cerca de 7 millones viven en Latinoamérica y el Caribe. La prevalencia de la epilepsia en Latinoamérica y el Caribe es mayor que la existente en los países desarrollados (1).

La definición de epilepsia del lóbulo temporal se estableció tras la lobectomía realizada por Penfield en 1954, posteriormente fue modificado por Walker en 1967 y Falconer en 1979 (2,3). La Comisión de Epidemiología y Pronóstico de la Liga Internacional contra la Epilepsia (ILAE) define a las crisis epilépticas como: "Manifestación clínica presumiblemente originada por una descarga excesiva de neuronas a nivel cerebral. Ésta consiste en fenómenos anormales bruscos y transitorios que incluye alteraciones de la conciencia, motoras, sensoriales, autonómicas, psicológicas, que son percibidos por el paciente o un observador" (4 - 8).

Entre los factores que podrían influir podemos mencionar la desnutrición, infecciones del sistema nervioso central, complicaciones del embarazo, parto y accidentes. Por otra parte, la prevalencia varía de un país a otro, incluso en distintas áreas de un mismo país (1).

El objetivo de este trabajo es exponer el protocolo básico de epilepsia y su utilidad en el planeamiento quirúrgico $\mathrm{y}$, de este modo, conociendo todas las herramientas estudiadas hasta el momento, se realice un oportuno diagnóstico para lograr un tratamiento adecuado y certero de estos pacientes.

\section{Anatomía y fisiopatología}

Nuevos métodos diagnósticos han permitido determinar la participación de esta región anatómica como generadora de la epilepsia; entre estos tenemos la resonancia magnética (RM), la tomografía computarizada craneal por emisión de fotón único (SPECT), la tomografía por emisión de positrones (PET) y la videomagnetoencefalografía (VMEEG).

El término epilepsia no se refiere a una enfermedad específica sino a un conjunto heterogéneo de trastornos neurológicos que representan una reacción anormal del cerebro a ciertos tipos de agresión (9). La manifestación clínica de la epilepsia son las crisis epilépticas. La gran mayoría de los casos de crisis corresponden a la epilepsia del lóbulo temporal (ELT), la cual es la forma más frecuente en los adultos, representando entre el $25 \%$ y $35 \%$ de los casos generales de epilepsia (10), cuya anormalidad anatómica más habitual es la esclerosis del hipocampo (11), seguida por tumores de bajo grado, malformaciones congénitas, vasculares, metabólicas, lesiones glióticas debidas a traumatismos o infecciones e idiopáticas que son las de peor pronóstico (12).

La fisiopatología de la esclerosis mesial temporal (EMT) presenta al menos dos factores comprometidos: la presencia de una lesión inicial debida a una enfermedad cerebral o médica en las etapas iniciales del desarrollo como convulsión febril prolongada, infección del sistema nervioso central o trauma, y como segundo factor la predisposición individual que aumenta la vulnerabilidad para lesión neuronal como disgenesia hipocampal, convulsiones previas y predisposición genética(13).

Después del daño hipocámpico inicial se produce una pérdida neuronal con reorganización sináptica en las fibras musgosas, astrogliosis (14) y la formación de circuitos axonales aberrantes que facilitan la presencia de mecanismos de excitotoxicidad y de sinaptogénesis reactiva que contribuyen a la cronicidad de las convulsiones (9). Las alteraciones son más graves en algunas zonas del hipocampo, el cual se encuentra dividido en cuatro regiones CA1- 4 
(Figura 1). La esclerosis clásica del hipocampo consiste, principalmente, en una notable pérdida de células en CA1 y en la capa polimórfica del giro dentado, seguido de las regiones $\mathrm{CA} 4$ y CA3, mientras que las neuronas de CA2 y las células granulares del giro dentado sobreviven $(15,16)$, existiendo una vulnerabilidad selectiva, no solamente en el cerebro sino dentro del propio hipocampo (17). Esta selectividad lesional se explica por fenómenos ultraestructurales los cuales se basan en que existe mayor densidad de receptores para glutamato en CA1, CA3 e hilus de la fascia dentada.

Es conocida la relación del glutamato con el fenómeno de excitocitosis; el estímulo de sus receptores produce influjo de calcio a las células involucradas con el consecuente efecto deletéreo. Por el contrario, se han encontrado proteínas fijadoras de calcio (calbindina y cromogranina A) en mayor concentración en CA2 y en células granulares de la fascia dentada, estando prácticamente ausentes en $\mathrm{CA} 1$ y CA3; al actuar como
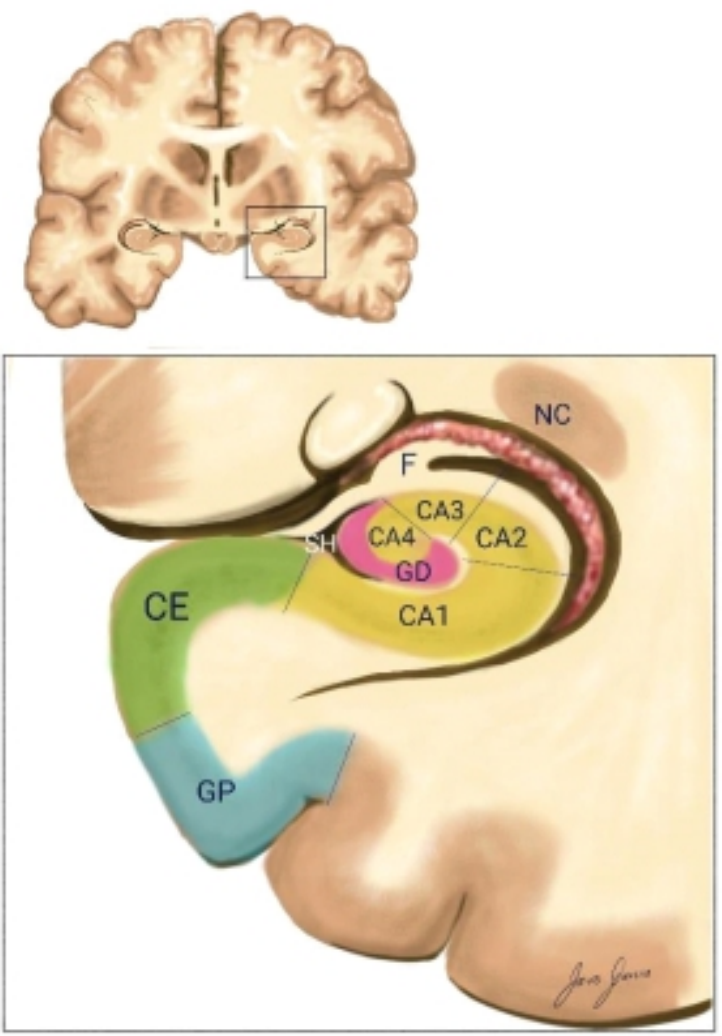

Figura 1. Anatomía del hipocampo, el cual se encuentra dividido en 4 regiones. NC: Núcleo caudado F: Fornix GD: Giro dentado CE: Corteza entorrinal GP: Giro parahipocampal.

Fuente: Elaborado por los autores. tampones para iones de calcio generan un efecto neuroprotector. Esto se traduce macroscópicamente en atrofia del hipocampo y de las estructuras adyacentes (amígdala, corteza entorrinal y giro parahipocampal (18). ILAE determinó una clasificación semicuantitativa en especímenes como ILAE tipo 1 con pérdida neuronal y gliosis predominantemente en CA1 y CA4, la afectación en el sector CA3 Y CA2 es variable; es el tipo más común, aproximadamente entre el $60 \%$ y el $80 \%$ se asocia frecuentemente con historia de lesión, se precipita antes de los 5 años, con inicio temprano de las convulsiones y control posquirúrgico favorable (19, 20), ILAE tipo 2 pérdida en CA1 y ILAE tipo 3 predominantemente en CA4, y sin esclerosis del hipocampo (21). Existe una clasificación neuropatológica para esclerosis mesial que clasifica la esclerosis hipocampo en tipo 1a donde CA1 y CA4 son los segmentos más comprometidos, tipo $1 \mathrm{~b}$ en donde existe pérdida neuronal severa en todos los segmentos del hipocampo y tipo 2 donde existe una pérdida neuronal severa restringido al segmento CA1 (22) y el tipo 3 donde existe significativa pérdida neuronal en todos los segmentos a excepción del segmento CA1 (23).

\section{Presentación clínica}

La semiología epileptogénica está influida por mecanismos relacionados con la edad. En los adultos se caracteriza por crisis estereotipadas que se manifiestan con dolor epigástrico, disminución de la actividad motora, mirada pérdida, alteración del estado de conciencia, automatismos en las manos, midriasis, cefalea, taquicardia y taquipnea; esto refleja la activación de las estructuras temporolímbicas y de los circuitos de la corteza prefrontal. En los niños las crisis no son tan homogéneas, sino que se observa gran variedad de las mismas presentando movimientos tónicos, clónicos, mioclónicos, hipermotores, espasmos, automatismos e inclusive un componente hipomotor, esto se debe a la madurez tardía del sistema límbico (2).

Desde el punto de vista clínico, los pacientes manifiestan antecedentes como convulsiones febriles, meningitis o encefalitis, traumatismo craneoencefálico, asfixia perinatal e historia familiar de epilepsia (24). En la mayoría de los casos los pacientes presentan períodos libres de crisis a lo largo de los años "luna de miel" y reinician las crisis cuando son adultos (24). Frecuentemente, al final de la primera o segunda década de la vida, el paciente se presenta con la primera crisis no febril, regularmente es una crisis parcial 
compleja, aunque puede ser parcial simple o generalizada.

Más del $80 \%$ de los pacientes con ELT mesial presentan auras (24) que, en la mayoría de las veces, se manifiestan como ansiedad, déjà vu o miedo, asociadas con síntomas viscerales, como náuseas y una sensación indescriptible que asciende desde el epigastrio (25). Después del aura, el paciente presenta mirada fija y paro motor, seguidos de automatismos oroalimentarios o manuales, sumados a manifestaciones autonómicas como midriasis, hiperventilación, piloerección y taquicardia (26). Ocasionalmente se puede detectar una postura distónica en el miembro superior contralateral a la zona de inicio ictal (27). Es posible encontrar alteraciones del lenguaje durante la crisis que sugieren un foco en el lóbulo temporal dominante, o alteraciones del lenguaje en el estado posictal que sugieren un foco del lóbulo temporal no dominante (26). Hasta un $50 \%$ de los pacientes pueden presentar crisis tonicoclónicas secundariamente generalizadas que no son homogéneas debido a la propagación eléctrica extensa a través de diferentes vías, entre las que se encuentran, probablemente, estructuras cerebrales profundas como los ganglios basales y tálamos (2).

\section{Diagnóstico imagenológico}

La resonancia magnética encefálica se considera el método diagnóstico estándar para la esclerosis temporal mesial porque presenta mayor sensibilidad (95\%), mejor resolución espacial, excelencia de los tejidos blandos, posibilidad de imágenes multiplicares y ausencia de radiación ionizante (28).

Se reconocen los signos primarios: un hipocampo pequeño o atrófico y un aumento en la intensidad de señal (Figura 2) $(29,30)$, ipsilaterales al foco epileptógeno, hallazgos que se correlacionan con la pérdida neural y la gliosis $(30,31)$.

Los signos secundarios incluyen pérdida de la arquitectura interna del hipocampo, pérdida de las digitaciones de la cabeza del hipocampo que normalmente contiene dos o tres digitaciones (Figura 3 izquierda), dilatación del asta temporal ipsilateral asociada a la atrofia hipocampal, aumento de la intensidad de señal de la amígdala, pérdida de volumen del lóbulo temporal (31), atrofia del cuerpo mamilar (Figura 3 derecha), atrofia del fórnix (Figura 4), atrofia del tálamo y atrofia del giro del cíngulo, ipsilateral a la afectación hipocampal (3234).

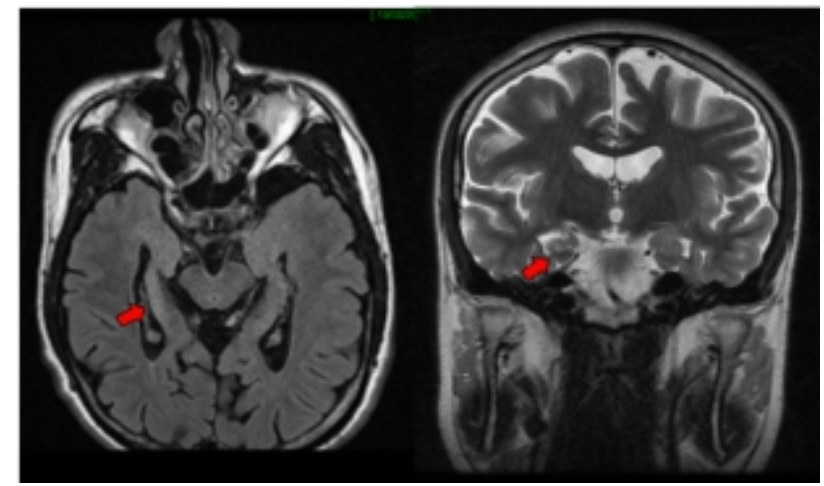

Figura 2. RMN Cerebral. Izquierda: Corte axial FLAIR. Hiperintensidad y disminución del tamaño del hipocampo derecho (Flecha recta). Derecha: Corte coronal T2. Hiperintensidad y disminución del tamaño del hipocampo derecho (Flecha recta).

Fuente: Elaborado por los autores.

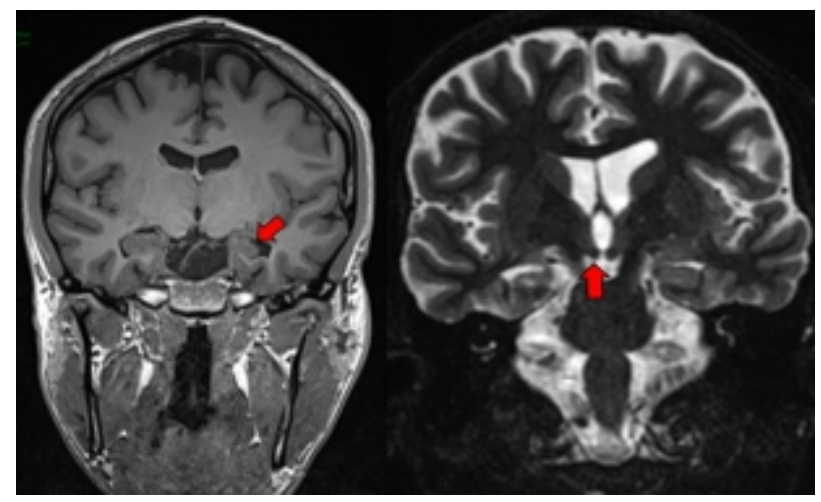

Figura 3. RMN Cerebral. Izquierda: Corte coronal T1. Pérdida de las digitaciones del hipocampo izquierdo (Flecha recta). Derecha: Corte coronal T2. Disminución del tamaño del cuerpo mamilar derecho (Flecha recta).

Fuente: Elaborado por los autores.

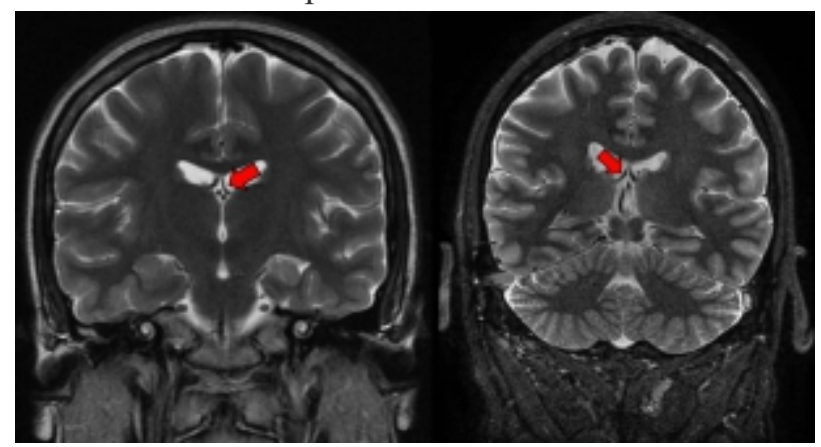

Figura 4. RMN Cerebral. Izquierda: Corte coronal T2 FSE. Asimetría del tamaño de los fórnix con disminución del tamaño del lado izquierdo (Flecha recta). Derecha: Corte coronal T2. Asimetría del tamaño de los fórnix con disminución del tamaño del lado derecho (Flecha recta).

Fuente: Elaborado por los autores. 
La ILAE recomienda realizar una RM a todo paciente con diagnóstico de epilepsia, salvo evidencia inequívoca de epilepsia generalizada idiopática o benigna de la infancia. También lo recomienda cuando las crisis comienzan en la edad adulta, cuando son de difícil control o han cambiado de patrón. La RM está indicada para los casos en que exista sospecha de focalidad, aunque existan estudios previos negativos. Además, deben realizarse controles periódicos en lesiones con potencial riesgo de crecimiento o sangrado, independientemente de la clínica. Durante el período de maduración cerebral pueden pasar desapercibidas algunas lesiones, razón por la cual se recomienda repetir el estudio entre los 24 y 30 meses una vez completada la mielinización (8). La Comisión en neuroimagen de la ILAE recomienda un protocolo específico de resonancia magnética en epilepsia (35).

Numerosos grupos de trabajos han probado que el empleo de imanes de campo inferior a $1.5 \mathrm{~T}$ está absolutamente contraindicado y que el uso de secuencias rutinarias es inadecuado porque disminuye significativamente el rendimiento de la prueba (35, 36).

El objetivo fundamental de un estudio estructural en pacientes con epilepsia es conseguir el máximo contraste entre la sustancia blanca (SB) y la sustancia gris ( $\mathrm{SG})$, además de trabajar con una alta resolución espacial, tanto en el propio corte (matriz de adquisición) como en el espesor del corte que debe oscilar entre los 0.5 - $1 \mathrm{~mm}$ de los estudios 3D y los 2 - 4 mm de los estudios 2D $(17,35,37)$.

Las secuencias $\mathrm{T} 1$ han de adquirirse en modo 3D, con voxel isotrópico de $1-1.5 \mathrm{~mm}$, incluyendo todo el cerebro. Generalmente se emplean secuencias de eco de gradiente (EG) con pulso de preparación en inversión recuperación para aumentar el contraste entre sustancia blanca y sustancia gris. Esta secuencia permite la reconstrucción en cualquier plano sin perder calidad de imagen $\mathrm{y}$, en caso necesario, realizar estudios volumétricos o reconstrucciones curvas. (30, $35,37)$.

En un estudio de epilepsia, es necesario hacer cortes coronales perpendiculares al hipocampo, porque son los que aportan más información. Se precisan imágenes de alta resolución en T2, con píxel inferior a $0.5 \mathrm{~mm}$, es decir que en un campo de visión (FOV) de $240 \mathrm{~mm}$ la matriz ha de ser de 512 o mayor. Se deben utilizar secuencias 2D-fast-SE con un espesor de corte de $3 \mathrm{~mm}$. Además, hay que añadir secuencias flatattenuated inversión recovery (FLAIR) en los mismos planos, buscando más una alteración en el contraste que una alteración en la forma o en la estructura interna del hipocampo. La resolución de los cortes puede ser menor, con matrices de 256 y espesor de corte de 3 - 4 $\mathrm{mm}$. Las secuencias FLAIR son más sensibles que las secuencias T2 para detectar pequeñas alteraciones de señal, especialmente en áreas adyacentes al líquido cefalorraquídeo $(30,36,37)$

Deben añadirse cortes axiales de $4-5 \mathrm{~mm}$ con secuencias $\mathrm{T} 2 *$, bien sea con técnica de $\mathrm{EG}$ convencional o con técnicas de eco-planar (EPI) más rápidas, que no necesitan matrices de alta resolución $(7,38)$.

Además, aunque exista una alta sospecha de que el origen sea temporal, hay que estudiar todo el cerebro con una secuencia axial FLAIR (opcionalmente T2) para descartar pequeñas lesiones que causen directamente las crisis, o asociadas a una alteración del hipocampo (lesión dual) (35 - 37).

En resumen, un protocolo básico de epilepsia incluye secuencias 3D-T1, cortes coroneles T2 y FLAIR, y cortes axiales FLAIR y T2*. La Utilización de Gadolinio no es requerida, solo se utiliza ante la sospecha de tumor, malformación arteriovenosa y síndromes neurocutáneos (37 - 41).

La espectroscopia es una técnica utilizada en equipos de alto teslaje que se obtiene con técnica univoxel, con tamaño del voxel de $10 \times 10 \times 25,2500 \mathrm{~mm}^{3}$ posicionado sobre el lóbulo mesotemporal incluyendo en hipocampo (Figura 5) (42). En esclerosis mesial temporal demuestra disminución del pico del NAA $(\mathrm{N}-$ Acetil Aspartato) y de los ratios NAA/Cho (Colina), NAA/Cr (Creatina) y NAA/ Cho-Cr en el lado patológico hasta en el $90 \%$ de los casos indican pérdida neuronal y disfunción. Sin embargo, este hallazgo también lo podemos observar en el lado contralateral en un $30 \%-40 \%$. La relación NAA/colina+creatinina es el parámetro más sensible para detectar la anormalidad funcional en el hipocampo afectado. Una reducción de este índice menor a 0.71 ppm (Figura 6) (43-47) y un índice de asimetría menor del $11 \%$ es patológico, lo que permite identificar la lateralización. Si la relación NAA/Colina +Creatina está afectada en los dos hipocampos el índice utilizado para lateralización es de $5.5 \%(43,44)$.

La volumetría del hipocampo puede ser de utilidad en el ámbito de la investigación y en los casos dudosos, especialmente cuando se sospeche una ETM bilateral 


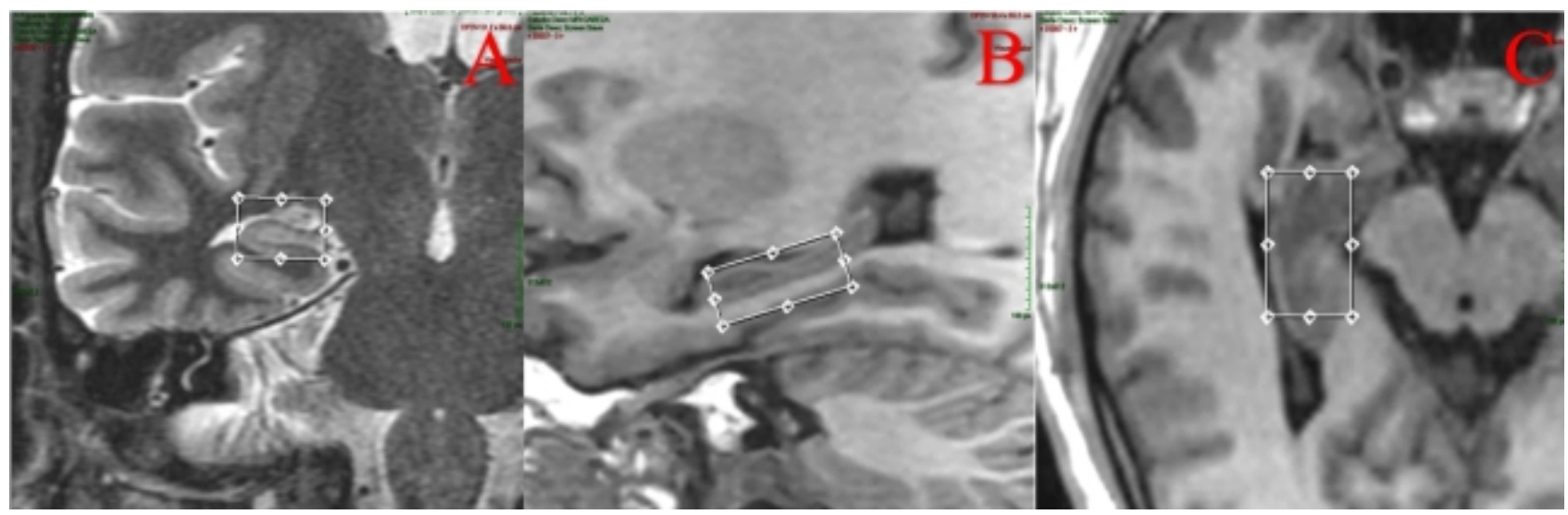

Figura 5. RMN Cerebral. Técnica de espectroscopía univoxel posicionado sobre el hipocampo. A. Coronal T2. B. Sagital T1. C.Axial T1.

Fuente: Elaborado por los autores.

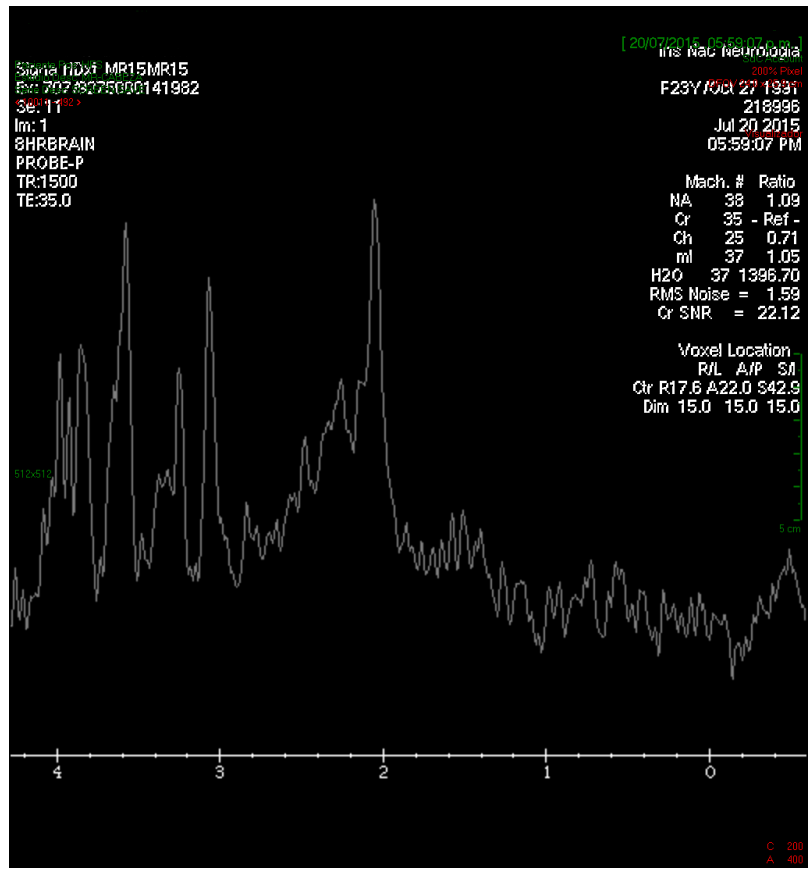

Figura 6. Espectroscopia. Relación NAA/ colina + creatinina $=0.63$ en el lado derecho.

Fuente: Elaborado por los autores.

en la que el análisis visual es más limitado. La evaluación visual puede detectar adecuadamente asimetrías hipocampales mayores al $20 \%$ (47); sin embargo, menores grados de asimetría requieren análisis cuantitativos. La reducción del volumen hipocampo se correlaciona con la severidad de pérdida neuronal $(47,48)$. El análisis cuantitativo del hipocampo se realiza mediante volumetría hipocampal $(45,49)$ de ambos hipocampos, calculando el índice volumétrico de asimetría (IVA), definido como la diferencia absoluta entre los volúmenes de cada hipocampo divididos por su promedio; este índice representa la metodología en uso para la interpretación de la volumetría hipocampal considerándose patológico cuando es mayor al $10 \%$ (45). Se obtienen imágenes volumétricas de alta resolución espacial con técnicas $3 \mathrm{D}$, los límites del hipocampo se definen de la siguiente manera: el límite anterior es el primer corte donde la amígdala no se ve, el límite lateral se traza a lo largo del cuerno temporal, en la parte superior el líquido cefalorraquídeo en la cisura coroidea, medialmente por el líquido cefalorraquídeo adyacente a la cisterna uncal y la cisterna ambiens, en la parte inferior la sustancia gris del giro parahipocampal (42), el límite posterior es inmediatamente anterior a la cruz del fornix (50). El análisis se puede realizar con técnicas semiautomatizadas $(42,51)$ o automatizadas, según los softwares disponibles en el mercado $(52,53)$.

La utilización de equipos de $3 \mathrm{~T}$ aumenta la relación señal/ruido en una proporción casi lineal al aumento del campo y, además, mejoran el contraste en T2, por lo tanto, es posible aumentar la resolución y el contraste para estudiar epilepsias refractarias $(29,47,54)$.

La resonancia magnética de ultra alto campo (mayor a 7T) permite la detección selectiva de anormalidades en los diferentes segmentos del cuerno deAmmon, ya que permite evaluar adecuadamente la anatomía con valoraciones individualizadas como la disminución de las dignaciones de la cabeza del hipocampo y detectar anomalías o atrofia de los diferentes segmentos del hipocampo, mejorando la detección de la esclerosis hipocampaly de displasia cortical focal. (55 - 57). 
Para planeamiento quirúrgico se han desarrollado técnicas imagenológicas como la tractografía y la resonancia magnética funcional que permiten mejor la caracterización de las lesiones y de la conectividad neuronal, y permiten una adecuada planificación prequirúrgica de las lesiones cerebrales cercanas a las áreas elocuentes.

La tractografía es la única técnica no invasiva que permite la disección in vivo de las fibras de la sustancia blanca. Se trata de una técnica basada en difusión por tensor que cuantifica la magnitud y dirección del movimiento microscópico de las moléculas de agua. Es útil para detectar alteraciones en la microestructura de los fascículos de sustancia blanca, pudiendo observar de manera cualitativa la pérdida de continuidad de las fibras o la superposición de anisotropía, direccionalidad y conectividad (58). En epilepsia del lóbulo temporal se puede evaluar la vía visual y predecir la ocurrencia y extensión de defectos del campo visual posterior a resección temporal anterior, evaluando la extensión anterior del asa de Meyer dentro del polo temporal, donde se ha demostrado que longitudes menores a 35 $\mathrm{mm}$ del aspecto anterior del asa de Meyer, se asocia a defectos de los campos visuales posquirúrgicos (59), también se pueden evaluar las radiaciones ópticas pre y posquirúrgicamente y predecir la extensión del defecto de los campos visuales (60).

La tractografía puede demostrar reorganización estructural de los tractos asociados a memoria y lenguaje de acuerdo con la localización de la lesión y a la dominancia hemisférica; en pacientes con dominancia hemisférica izquierda y esclerosis temporal mesial ipsilateral se ha demostrado aumento de los tractos de sustancia blanca del hemisferio contralateral como resultado de reorganización funcional del lenguaje en regiones corticales contralaterales $(61,62)$. $\mathrm{La}$ evaluación del fascículo arcuato que conecta las áreas receptivas y productivas del lenguaje ha demostrado ser mayor en el hemisferio dominante y esa asimetría se reduce cuando existe lesión en el hemisferio dominante secundaria a reorganización funcional $(63,64)$, mientras que la evaluación del fascículo uncinado (Figura 7) y los datos de reorganización neuronal permiten predecir el desarrollo de afasia (65).

La resonancia magnética funcional se basa en el aumento del flujo sanguíneo en áreas neurológicamente activadas donde disminuyen los niveles de desoxihemoglobina, la cual es una sustancia paramagnética y aumenta la intensidad de señal en secuencias de T2 específicamente en secuencias BOLD (imagen dependiente del nivel de oxígeno sanguíneo, permitiendo el mapeo de funciones cerebrales basados en los cambios hemodinámicos cerebrales que corresponden a una tarea específica) $(18,66,67)$, lo cual permite la evaluación prequirúrgica para pacientes con epilepsia refractaria y candidatos a cirugía del lóbulo temporal, para planeamiento quirúrgico y balance de riesgos y beneficios de la cirugía $(47,66)$.

La resonancia magnética funcional en pacientes con epilepsia del lóbulo temporal secundaria a esclerosis temporal mesial permite delinear áreas del cerebro responsables de funciones específicas como áreas del lenguaje y memoria, identificando su localización

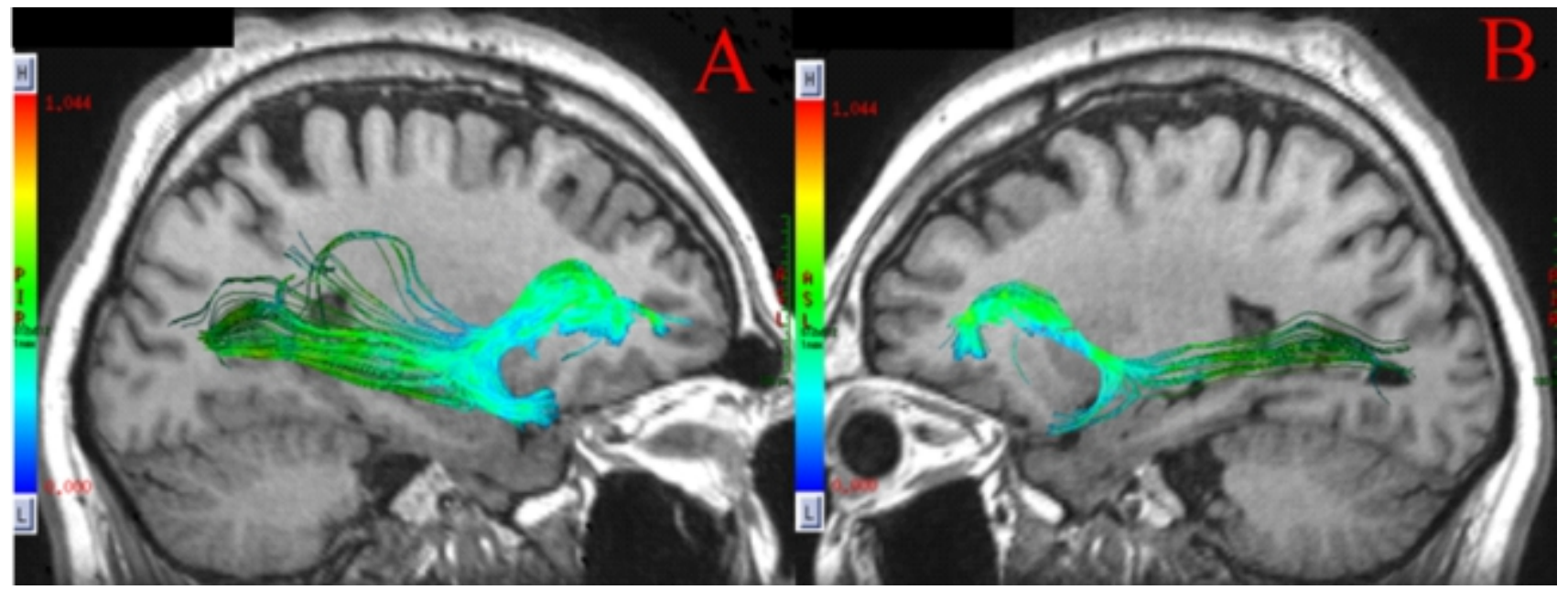

Figura 7. RMN Cerebral. Tractografía. Corte sagital. A. Fascículo uncinado derecho normal. B. Fascículo uncinado izquierdo anormal con disminución de su calibre y alteración de la arquitectura.

Fuente: Elaborado por los autores. 


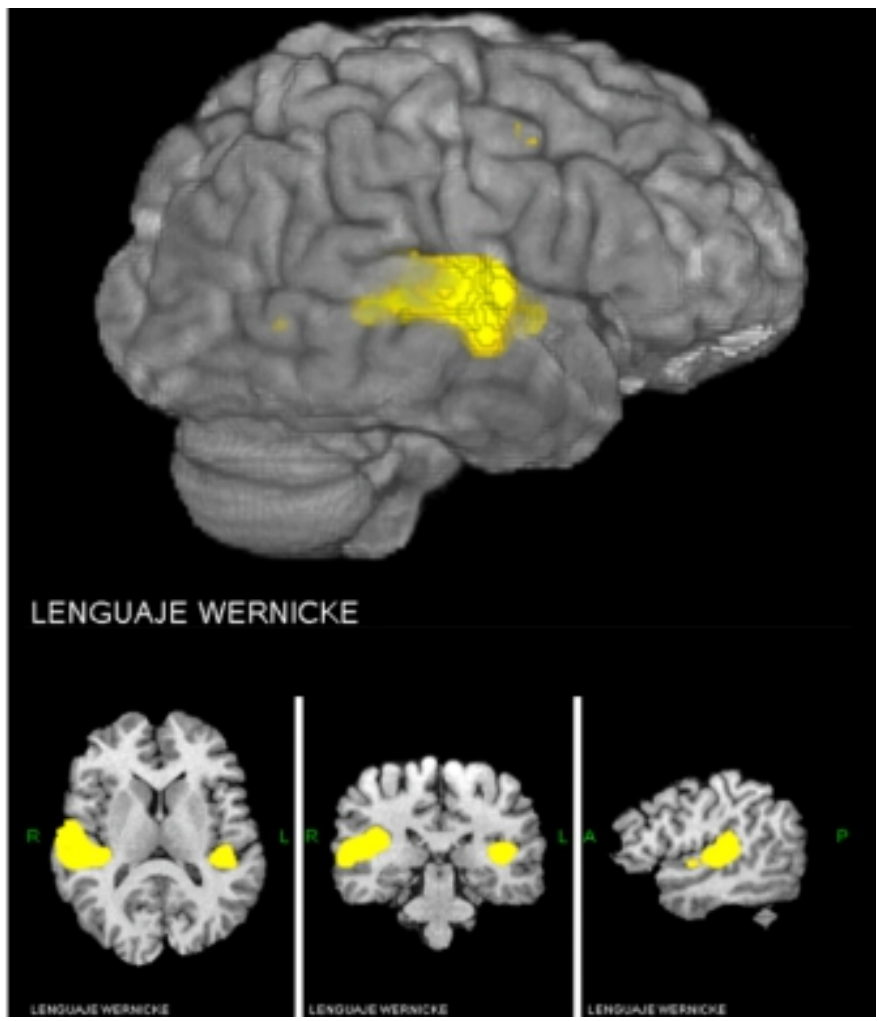

Figura 8. RMN Cerebral funcional. Área asociada a funciones de procesamiento de lenguaje receptivo del giro temporal medio y superior (áreas 21 y 22, Wernicke).

Fuente: Elaborado por los autores.

anatómica para mejor planeamiento quirúrgico y anticipación de déficit cognitivos postquirúrgicos (47, 66). La lateralización de las funciones del lenguaje con resonancia magnética funcional presenta buena correlación con el test carotideo con amobarbital (Test de WADA) (67 - 70). Pacientes con epilepsia del lóbulo temporal del lado izquierdo muestran activación bilateral o lateralización hemisférica derecha, o patrones atípicos de dominancia del lenguaje en relación con la reorganización en la representación del lenguaje $(61,66,71)$. Utilizando áreas de comprensión lectora para activación del giro temporal superior (Figura 8) y tareas de fluencia verbal y generación de verbos para activación de giro frontal inferior, se logra lateralizar la función del lenguaje y ofrecer datos para mejor planeamiento quirúrgico de lesiones del hemisferio dominante (68, 72, 73).

La evaluación de funciones de memoria requiere la utilización de paradigmas específicos que permitan activaciones congruentes para poder predecir déficits posquirúrgicos $(66,74$ - 76). Para lograr una adecuada activación del hipocampo del hemisferio dominante, generalmente el izquierdo, se requieren tareas de memoria verbal, y para activación del hipocampo no dominante derecho se utilizan tareas de memoria visual (77), que, entre más anterior se localizan las áreas de activación, mayor déficit pueden predecir; a diferencia de activaciones más posteriores que son resultado de reorganización de la función, que se asocian a mejor preservación de las funciones de memoria(78).

El estudio del estado de reposo cerebral Resting State a través de técnicas de resonancia magnética funcional permite el análisis de la actividad cerebral; cuando el paciente está en reposo sin una actividad mental específica la red neuronal que se activa durante el reposo se conoce como circuito de activación por defecto, que revela patrones de actividad cortical sincronizados, lo que permite conocer la arquitectura funcional intrínseca del cerebro (79 -83). Esto se ha utilizado para investigar alteraciones de la red del hipocampo y su relación con la duración de la epilepsia en pacientes con esclerosis temporal. La conectividad hipocampal interhemisférica se interrumpe inicialmente, luego aumenta linealmente con el 
hipocampo contralateral al foco epileptogénico que influye en el hipocampo ipsilateral (66). Recientes estudios han demostrado alteraciones en los circuitos de activación por defecto en los circuitos de atención y en los de control emocional, sugiriendo que la esclerosis temporal mesial modifica crónicamente la actividad de los circuitos del cerebro para el control de funciones básicas (84). Adicionalmente, se ha identificado una región en el núcleo ventral lateral del tálamo derecho cuya conectividad con el hipocampo separa a los sujetos con esclerosis temporal izquierda de derecha. Esto sugiere que la cuantificación de la conectividad funcional de estado de reposo a través de esta red puede ser un indicador potencial de lateralización de esclerosis temporal mesial que se puede añadir a otras evaluaciones prequirúrgicas (84), lo cual requiere nuevas investigaciones que lo valiesen, poniendo en evidencia la gran potencialidad de esta técnica aún en desarrollo.

\section{Conclusiones}

La epilepsia del lóbulo temporal es el tipo de epilepsia focal más frecuente en adultos, se caracteriza por tener el foco epileptogénico localizado en los lóbulos temporales, con signos y síntomas característicos que orientan el diagnóstico, y una fisiopatología cada vez más conocida que permite un adecuado control de las crisis. Generalmente los pacientes tienen antecedentes de convulsiones febriles en la infancia, meningitis, asfixia perinatal e historia familiar de epilepsia, y al final de la primera o segunda década de la vida presentan la primera crisis no febril asociada a auras.

En caso de no lograr control farmacológico de la crisis, presenta una alta probabilidad de control con técnicas quirúrgicas cada vez menos agresivas; es aquí donde las imágenes juegan un papel muy importante como ayuda para el cirujano. El hecho de sospechar clínicamente, solicitar y conocer todos los avances y las técnicas estructurales y funcionales expuestas anteriormente basadas en imágenes tipo resonancia magnética nuclear, permiten realizar un adecuado y oportuno diagnóstico que a largo plazo disminuye la morbimortalidad de estos pacientes.

\section{Consideraciones éticas}

Todas las imágenes radiológicas tienen consentimiento informado escrito y su registro fue realizado por el médico radiólogo a cargo de cada caso.

\section{Conflicto de intereses}

Los autores declaran que no existen conflictos de intereses.

\section{Referencias}

1. Acevedo C, Miranda C, Campos M, Caraballo R, Carpio A, Cuadra L, et al. Informe sobre la epilepsia en Latinoamérica [Internet]. Organización Panamericana de la Salud; 2008 [citado 15 de septiembre de 2018]. 92 p. Recuperado a partir de: http://new.paho.org/hq/ dmdocuments/2008/Informe_sobre_epilepsia.pdf.

2. Volcy Gómez M. Mesial temporal lobe epilepsy: its physiopathology, clinical characteristics, treatment and prognosis. Revista de neurología. 2004 1;38(7):663.

3. Walker AE. Temporal lobectomy. J Neurosurg. $1967 ; 26: 642$. d o i : https://doi.org/10.3171/jns.1967.26.6.0642

4. Commission on Classification and Terminology of the International League Against Epilepsy. Proposal for revised clinical and electroencephalographic classification of epileptic seizures. Epilepsia. 1981;22:489-501. doi: 10.1111/j.1528-1157.1981.tb06159.x

5. Commission on Classification and Terminology of the International League against Epilepsy. Proposal for classification of epilepsies and epileptic syndromes. Epilepsia. 1985;26(3):268. doi: https://doi.org/10.1111/j.1528-1157.1985. tb05417.x

6. Commission on Classification and Terminology of the International League Against Epilepsy. 1989. Proposal for revised classification of epilepsies and epileptic syndromes. Epilepsia. 1989;30(4):389399. doi: https://doi.org/10.1111/j.15281157.1989.tb05316.x

7. Engel J. ILAE classification of epilepsy syndromes. Epilepsy research. 2006;70 Suppl 1:S5-10. doi: 10.1016/j.eplepsyres.2005.11.014

8. Berg AT, Berkovic SF, Brodie MJ, Buchhalter J, Cross JH, Emde WV et al. Revised terminology and concepts for organization of seizures and epilepsies: Report of the ILAE Commission on Classification and Terminology, 2005-2009. Epilepsia. 2010;51(4):676-85. doi: 10.1111/j.1528-1167.2010.02522.x.

9. Pereno G. Fisiopatología de la Epilepsia del Lóbulo Temporal: Revisión del Proceso de Muerte neuronal a la neuroplasticidad. Revista Argentina de Ciencias del Comportamiento. 2010 ; Vo $1.2, \mathrm{~N}^{\circ} 1,46-57$. do i : http://dx.doi.org/10.32348/1852.4206.v2.n1.5269.

10. McHugh JC, Delanty N. Epidemiology and 
classification of epilepsy: gender comparisons. Int Rev Neurobiol. 2008;83:11-26. doi: 10.1016/S0074-7742(08)00002-0.

11. Sloviter R.S. Hippocampal epileptogenesis in animal models of mesial temporal lobe epilepsy with hippocampal sclerosis: the importance of the "latent period" and other concepts. Epilepsia. 2008;49 Suppl 9:85-92. doi: 10.1111/j.15281167.2008.01931.x

12. Cendes F. Mesial temporal lobe epilepsy syndrome: an updated overview. J Epilepsy Clin Neurophysiol. 2005;11(3):141-144. doi: http://dx.doi.org/10.1590/S 1676 26492005000300006.

13. Lewis DV. Losing neurons: selective vulnerability and mesial temporal sclerosis. Epilepsia. 2005;46 Supp1 7(s7):39-44. doi: 10.1111/j.15281167.2005.00306.x

14. Mathern GW, Babb TL, Vickrey BG, Melendez M, Pretorius JK. The clinical-pathogenic mechanisms of hippocampal neuron loss and surgical outcomes in temporal lobe epilepsy. Brain. 1995;118 (Pt 1):105-18. doi: 10.1093/brain/118.1.105

15. Oroquieta JF, Arellano J.I, Alonso L, Muñoz A. Neuropatología de la epilepsia del lóbulo temporal. Alteraciones primarias y secundarias de los circuitos corticales y epileptogenicidad. Rev Neurol. 2002;34(5):401-408. doi: https://doi.org/10.33588/rn.3405.2001403.

16. Pastor J, Uzcátegui Y. G, Gal-Iglesias D, Ortega G. E, Sola R, Menéndez de la Prida L. Bases fisiopatológicas de la epilepsia del lóbulo temporal: estudios en humanos y animales. Rev Neuro1. 2006;42(11):663-673. doi: https://doi.org/10.33588/rn.4211.2006142

17. Álvarez J. Resonancia magnética estructural en la epilepsia. Radiología. 2010;54(1):9-20. doi: 10.1016/j.rx.2011.07.007

18. Vázquez $\mathrm{C}$, et al. Esclerosis temporal mesial: Paradigma de la epilepsia de resolución quirúrgica. Rev Argent Neuroc. 2008,22:169

19. Blumcke I, Thom M, Aronica E, Armstrong DD, Bartolomei F, Bernasconi A, et al. International consensus classification of hippocampal sclerosis in temporal lobe epilepsy: A Task Force report from the ILAE Commission on Diagnostic Methods. Epilepsia. 2013;54(7):1315-29. doi: 10.1111/epi.12220.

20. Blümcke I; Coras R; Miyata H; Özkara C. Defining Clinico-Neuropathological Subtypes of Mesial Temporal Lobe Epilepsy with Hippocampal Sclerosis. Brain Pathol. 2012;22(3):402-11. doi: 10.1111/j.1750-3639.2012.00583.x.

21. Elkommos S, Weber B, Niehusmann P, Volmering E, Richardson MP, Goh YY, et al. Hippocampal internal architecture and postoperative seizure outcome in temporal lobe epilepsy due to hippocampal sclerosis. Seizure. 2016;35:65-71. doi: 10.1016/j.seizure.2016.01.007

22. Prada A, Scarpa R, Sales LO, Penteado C, Murilo M, Silva R. Temporal lobe epilepsy with mesial temporal sclerosis: hippocampal neuronal loss as a predictor of surgical outcome. Arq Neuropsiquiatr. 2012;70(5):319-24. doi: http://dx.doi.org/10. 1590/S0004-282X2012000500003.

23. Blümcke I, Pauli E, Clusmann H, Schramm J, Becker A, Elger C, et al. A new clinicopathological classiWcation system for mesial temporal sclerosis. Acta Neuropathol. 2007;113(3):235-44. doi: 10.1007/s00401-0060187-0

24. Téllez JF, Ladino L. Epilepsia temporal: aspectos clínicos, diagnósticos y de tratamiento. Rev Neurol. $2013 ; 56(4): 229-242$. doi: https://doi.org/10.33588/rn.5604.2012661

25. So EL. Value and Limitations of Seizure Semiology in Localizing Seizure Onset. J Clin Neurophysiol. 2006;23(4):353-7. doi: 10.1097/01.wnp.0000228498.71365.7b

26. Tatum WO. Mesial temporal lobe epilepsy. J Clin Neurophysiol. 2012;29(5):356-65. doi: $10.1177 / 107385840100700410$

27. Maillard L, Vignal JP, Gavaret M, Guye M, Biraben A, McGonigal A, et al. Semiologic and electrophysiologic correlations in temporal lobe seizure subtypes. Epilepsia. 2004;45(12):1590-9. doi: 10.1111/j.0013-9580.2004.09704.x

28. Vattipally VR, Bronen RA. MR imaging of epilepsy: strategies for successful interpretation. Neuroimaging Clinics. 2004; Vol 14, Issue 3, 349 372. doi: https://doi.org/10.1016/j.nic.2004.04.002

29. Sawaishi Y, Sasaki M, Yano T, Hirayarma A, Akabane J, Takada G. A hippocampal lesion detected by high-field 3 tesla magnetic resonance imaging in a patient with temporal lobe epilepsy. Tohoku J Exp Med. 2005;205(3):287-91. doi: https://doi.org/10.1620/tjem.205.287

30. Isnard J, Bourdillon P. Morphological imaging of the hippocampus in epilepsy. Rev Neurol (Paris). $2015 ; 171$ ( 3 ): $298-306$. d o i : 10.1016/j.neurol.2014.12.002.

31. Ochoa-Escudero M, Herrera DA, Vargas SA, Dublin AB, MBA, FACR. Congenital and Acquired Conditions of the Mesial Temporal Lobe: A Pictorial Essay. Can Assoc Radiol J. $2015 ; 66(3): 238-51$. d o i : 10.1016/j.carj.2014.12.006.

32. Khashper A, Chankowsky J, del CarpioO'Donovan R. Magnetic Resonance Imaging of the Temporal Lobe: Normal Anatomy and Diseases. 
Canadian Association of Radiologists Journal. $2014 ; 65$ ( 2 ): $148-157$. d o i : https://doi.org/10.1016/j.carj.2013.05.001

33. Malmgren K, Thom M. Hippocampal sclerosis-Origins and imaging. Epilepsia. 2012;53 Suppl 4:19-33. doi: 10.1111/j.15281167.2012.03610.x

34. Cendes F. Neuroimaging in Investigation of Patients with Epilepsy. American Academy of Neurology. Continuum (Minneap Minn). $2013 ; 19$ (3 Epilepsy):623-42. doi: 10.1212/01.CON.0000431379.29065.d3

35. ILAE Commission Report. Recommendations for Neuroimaging of Patients with Epilepsy. Epilepsia. 1997;38(11):1255-6. doi: https://doi.org/ 10.1111/j.1528-1157.1997.tb01226.x

36. Oertzen JV, Urbach H, Jungbluth S, Kurthen M, Reuber M, Fernández G, et al. Standard magnetic resonance imaging is inadequate for patients with refractory focal epilepsy. J Neurol Neurosurg Psychiatry. 2002;73(6):643-7. doi: 10.1136/jnnp.73.6.643

37. Kuzniecky RI. Neuroimaging of Epilepsy: Therapeutic Implications. NeuroRx. 2005;2(2): 384-393. doi: 10.1602/neurorx.2.2.384

38. Vezina LG. MRI-negative epilepsy: Protocols to optimize lesion detection. Epilepsia. 2011;52 Suppl 4:25-7. doi: 10.1111/j.1528-1167.2011.03147.x

39. Kilpatrick C, O'Brien T, Matkovic Z, Cook M, KayeA. Preoperative evaluation for temporal lobe surgery. J Clin Neurosci. 2003;10(5):535-9. https://doi.org/10.1016/S0967-5868(03)00080-8

40. Friedman E. Epilepsy Imaging in Adults: Getting It Right. AJR Am J Roentgenol. 2014;203(5):1093103. doi: $10.2214 /$ AJR.13.12035

41. Ramli N, Rahmat K, Lim KS, Tan CT. Neuroimaging in refractory epilepsy. Current practice and evolving trends. Eur J Radiol. $2015 ; 84(9): 1791-800$. d o i : 10.1016/j.ejrad.2015.03.024

42. Lopez ML, Martinez M, Favila R, Roldan E. Secondary MRI-findings, volumetric and spectroscopic measurements in mesial temporal sclerosis: A multivariate discriminant analysis. Swiss Med Wkly. 2012;142:w13549 doi: 10.4414/smw.2012.13549

43. Brandao LA, Domingues RC. MR Spectroscopy of the Brain. Philadelphia, PA: Lippincott Williams \& Wilkins, 2004.

44. Achten E, Santens P, Boon P, Coo D, Kerckhove T, Reuck J, et al. Single-Voxel Proton MR Spectroscopy and Positron Emission Tomography for Lateralization of Refractory Temporal Lobe Epilepsy. AJNR Am J Neuroradiol [Internet]. 1998 [citado 15 de septiembre de 2018];19(1):1-8.
R e c u p e r a d o a partir d e : http://www.ajnr.org/content/19/1/1.long

45. Roldan E, Corona R, Cosme J, Martínez M. Esclerosis temporal mesial en epilepsia del lóbulo temporal: evaluación cuantitativa con resonancia magnética 3.0 Tesla. Gac Méd Méx [Internet]. 2007[citado 15 de septiembre de 2018]; Vol.143 No.5. Recuperado a partir de: https://www.medigraphic.com/pdfs/gaceta/gm2007/gm075o.pdf

46. Aydin H, Oktay N, Kizilgoz V, Altin E, Tatar I, Hekimoglu B. Value of Proton-MR-Spectroscopy in the Diagnosis of Temporal Lobe Epilepsy; Correlation of Metabolite Alterations with Electroencephalography. Iran J Radiol. 2012;9(1):1-11. doi: 10.5812/iranjradiol.6686.

47. Salmenpera TM, Duncan JS. Imaging In Epilepsy. J Neurol Neurosurg Psychiatry. 2005;76 Suppl 3:210. doi: http://dx.doi.org/10.1136/jnnp. 2005.075135

48. Marchetti RL, Bottino CM, Azevedo D, Nagahashi SK, Castro CC. Confiabilidade de medidas volumétricas de estruturas temporais mesiais. Arquivos de Neuro-Psiquiatria. 2002;60(2B):420428. doi: https://dx.doi.org/10.1590/S0004282X2002000300016

49. Paesschen WV. Qualitative and quantitative imaging of the hippocampus in mesial temporal lobe epilepsy with hippocampal sclerosis. Neuroimaging Clin N Am. 2004;14(3):373-400, vii. doi: 10.1016/j.nic.2004.04.004

50. Watson C, Andermann F, Gloor P, Jones-Gotman M, Peters T, Evans A, et al. Anatomic basis of amygdaloid and hippocampal volume measurement by magnetic resonance imaging. Neurology. 1992;42(9):1743-50. doi: 10.1016/j.nic.2004.04.004

51. Chee MW, Low S, Tan JS, Lim W, Wong J. Hippocampal Volumetry with Magnetic Resonance Imaging: A Cost-Effective Validated Solution. Epilepsia. 1997;38(4):461-465. doi: https://doi.org/10.1111/j.1528 1157.1997.tb01736.x

52. Farid N, Girard hm, Kemmotsu N, Smith ME, Magda SW, Lim WY, et al. Temporal lobe epilepsy: quantitative MR volumetry in detection of hippocampal atrophy. Radiology. $2012 ; 264(2): 542-50$. do i : 10.1148/radiol.12112638.

53. Bonilha L, Keller SS. Quantitative MRI in refractory temporal lobe epilepsy: relationship with surgical outcomes. Quant Imaging Med Surg. 2015;5(2):204-24. doi: 10.3978/j.issn.22234292.2015.01.01.

54. Hanamiya M, Korogi Y, Kakeda S, Ohnari N, 
Kamada K, Moriya J, et al. Partial loss of hippocampal striation in medial temporal lobe epilepsy: pilot evaluation with high-spatialresolution T2-weighted MR imaging at 3.0 T. Radiology. 2009;251(3):873-881. doi: doi: 10.1148/radiol.2513080445.

55. Henry TR, Chupin M, Lehéricy S, Strupp JP, Sikora MA, Sha ZY, et al. Hippocampal Sclerosis in Temporal Lobe Epilepsy: Findings at $7 \mathrm{~T}$. Radiology. 2011;261:199-209. doi: 10.1148/radiol.11101651.

56. Thomas BP, Welch EB, Niederhauser BD, Whetsell WO, Anderson AW, Gore JC, et al. HighResolution 7T MRI of the Human Hippocampus In Vivo. J Magn Reson Imaging. 2008;28(5):126672. doi: 10.1002/jmri.21576.

57. De Ciantis A, Barba C, Tassi L, Cosottini M, Tosetti M, Costagli M, et al. 7T MRI in focal epilepsy with unrevealing conventional field strength imaging. Epilepsia. 2016;57(3):445-54. doi: 10.1111/epi.13313.

58. Eriksson SH, Symms MR, Rugg-Gunn FJ, Boulby PA, Wheeler-Kingshott CA, Barker GJ, et al. Exploring white matter tracts in band heterotopia using diffusion tractography. Ann Neurol. 2002;52(3):327-34. doi: 10.1002/ana.10295.

59. Yogarjah M, Focke NK, Bonelli S, Cercignani M, Acheson J, Parer JM, et al. Defining Meyer's looptemporal lobe resections, visual field deficits and diffusion tensor tractography. Brain. 2009; 132(6): 1656-1668. doi: 10.1093/brain/awp114

60. Chen X, Weigel D, Ganslandt O, Buchfelder M, Nimsky C. Prediction of visual field deficits by diffusion tensor imaging in temporal lobe epilepsy surgery. Neuroimage. 2009;45(2):286-97. doi: 10.1016/j.neuroimage.2008.11.038.

61. Powell HW, Parker GJ, Alexander GC, Symms MR, Boulby PA, Wheeler-Kingshott CA, et al. Abnormalities of language networks in temporal lobe epilepsy. Neuroimage. 2007;36(1):209-21. doi: 10.1016/j.neuroimage.2007.02.028

62. Stylianou P, Hoffmann C, Blat I, Harnof S. Neuroimaging for patient selection for medial temporal lobe epilepsy surgery: Part 1 Structural neuroimaging. J Clin Neurosci. 2016;23:14-22. doi: 10.1016/j.jocn.2015.04.019.

63. Glasser MF, Rilling JK. DTI Tractography of the Human Brain's Language Pathways. Cereb Cortex. $2008 ; 18(11)$ : $2471-82$. d o i : 10.1016/j.jocn.2015.04.019

64. Lee DH, Hong CP, Kwon YH, Hwang YT, Kim JH, Park JW. Curvature range measurements of the arcuate fasciculus using diffusion tensor tractography. Neural Regen Res. 2013; 8(3): 244-250. doi: $10.3969 /$ j.issn. 1673 -
5374.2013.03.006

65. For SJkel, Thiebaut M, Dell'Acqua F, Kalra L, Murphy DG, Williams SC, et al. Anatomical predictors of aphasia recovery: a tractography study of bilateral perisylvian language networks. Brain. 2014;137(Pt 7):2027-39. doi: 10.1093/brain/awu113.

66. Stylianou P, Hoffmann C, Blat I, Harnof S. Neuroimaging for patient selection for medial temporal lobe epilepsy surgery: Part 2 functional neuroimagin. J Clin Neurosci. 2016;23:23-33. doi: 10.1016/j.jocn.2015.04.031.

67. Abou-Khalil B. An Update on Determination of Language Dominance in Screening for Epilepsy Surgery: The Wada Test and Newer Noninvasive Alternatives. Epilepsia. 2007;48(3):442-55. doi: 10.1111/j.1528-1167.2007.01012.x

68. Gaillard WD, Balsamo L, Xu B, et al. Language dominance in partial epilepsy patients identified with an fMRI reading task. Neurology. 2002;59(2):256-65. doi: 10.1212/wnl.59.2.256

69. Adcock JE, Wise RG, Oxbury JM, Oxbury SM, Matthews PM. Quantitative fMRI assessment of the differences in lateralization of language-related brain activation in patients with temporal lobe epilepsy. Neuroimage. 2003;18(2):423-38. doi: https://doi.org/10.1016/S1053-8119(02)00013-7

70. Binder JR, Swanson SJ, Hammeke TA, Morris GL, Mueller WM, Fischer M, et al. Determination of language dominance using functional MRI: A comparison with the Wada test. Neurology. 1996;46(4):978-84. doi: 10.1212/wnl.46.4.978

71. Thivard L, Hombrouck J, du Montcel ST, Delmaire C, Cohen L, Samson S, et al. Productive and perceptive language reorganization in temporal lobe epilepsy. Neuroimage. 2005;24(3):841-51. doi: 10.1016/j.neuroimage.2004.10.001

72. Metternich B, Buschmann F, Wagner K, SchulzeBonhage A, Kriston L. Verbal Fluency in Focal Epilepsy: A Systematic Review and Meta-analysis. Neuropsychol Rev. 2014;24(2):200-18. doi: 10.1007/s11065-014-9255-8.

73. Sanjuán A, Bustamante JC, Forn C, VenturaCampos N, Barrós-Loscertales A, Martínez JC, et al. Comparison of two fMRI tasks for the evaluation of the expressive language function. Neuroradiology. 2010;52(5):407-15. doi: 10.1007/s00234-010-0667-8.

74. Giovagnoli AR, Erbetta A, Villani F, Avanzini G. Semantic memory in partial epilepsy: verbal and non-verbal deficits and neuroanatomical relationships. Neuropsychologia. $2005 ; 43(10): 1482-92$. do i : 10.1016/j.neuropsychologia.2004.12.010

75. Richardson MP, Strange BA, Duncan JS, Dolan 
RJ. Memory fMRI in left hippocampal sclerosis: Optimizing the approach to predicting postsurgical memory. Neurology. 2006;66(5): 699-705. doi: 10.1212/01.wnl.0000201186.07716.98

76. Powell HW, Richardson MP, Symms MR, Boulby PA, Thompson PJ, Duncan JS, et al. Preoperative fMRI predicts memory decline following anterior temporal lobe resection. J Neurol Neurosurg Psychiatry. 2008;79(6):686-93. doi: 10.1136/jnnp.2007.115139

77. Sidhu MK, Stretton J, Winston GP, Symms M, Thompson PJ, Koepp MJ, et al. Memory fMRI predicts verbal memory decline after anterior temporal lobe resection. Neurology.

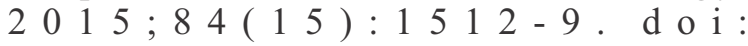
10.1212/WNL.0000000000001461.

78. Bonelli SB, Thompson PJ, Yogarajah M, Powell RH, Samson RS, McEvoy AW, et al. Memory reorganization following anterior temporal lobe resection: a longitudinal functional MRI study. Brain. 2013;136(Pt 6):1889-900. doi: 10.1093/brain/awt105.

79. Lee MH, Smyser CD, Shimony JS. Resting-State fMRI: A Review of Methods and Clinical Applications. AJNR Am J Neuroradiol. 2013;34(10):1866-72. doi: 10.3174/ajnr.A3263.

80. Proal E, Alvarez M, De la Iglesia M, Martí L,
Castellanos F. Actividad funcional cerebral en estado de reposo: Redes en conexion. Rev Neurol. $2011 ; 52\left(\begin{array}{ll}0 & 1\end{array}\right)$ : S $3-10$. d o i : https://doi.org/10.33588/rn.52S01.2010792

81. Barkhof F, Haller S, Rombouts SA. Resting-State Functional MR Imaging: A New Window to the Brain. Radiology. 2014;272(1):29-49. doi: 10.1148/radiol.14132388

82. Abela E, Rummel C, Hauf M, Weisstanner C, Schindler K, Wiest R. Neuroimaging of Epilepsy: Lesions, Networks, Oscillations. Clin Neuroradiol. 2014;24(1):5-15. doi: 10.1007/s00062-014-0284-8.

83. Cataldi M, Avoli M, de Villers-Sidani E. Resting state networks in temporal lobe epilepsy. Epilepsia. 2013;54(12):2048-59. doi: 10.1111/epi.12400.

84. Morgan VL, Sonmezturk HH, Gore JC, AbouKhalil B. Lateralization of temporal lobe epilepsy using resting functional Magnetic Resonance Imaging connectivity of hippocampal networks. Epilepsia. 2012;53(9):1628-35. doi: 10.1111/j.1528-1167.2012.03590.x. 